\title{
Determinação de fenol urinário por cromatografia em fase gasosa em trabalhadores que utilizam resinas fenólicas em fundições
}

\author{
Tiago Severo Peixe*, Elizabeth de Souza Nascimento, Henrique Vicente Della Rosa \\ Departamento de Análises Clínicas e Toxicológicas, Faculdade de Ciências Farmacêuticas, Universidade \\ de São Paulo
}

*Correspondência:

T. S. Peixe

Laboratório de Análises Clínicas e

Toxicológicas

Faculdade de Ciências Farmacêuticas

- USP

Av. Prof. Lineu Prestes, 580, BI 13B

05508-900 - São Paulo, SP - Brasil

E-mail: tpeixe@usp.br

\begin{abstract}
O fenol é utilizado na indústria como agente desinfetante no preparo de resinas fenólicas e pigmentos de tintas. Apresenta-se no estado sólido à temperatura ambiente, com coloração fracamente rósea, odor acre e higroscópico. Na exposição ocupacional aguda o composto pode levar a lesões eritematosas e, cronicamente, afetar a maturação celular no compartimento medular ósseo devido à formação de quinonas livres e 1,4-benzoquinona, proveniente do metabolismo hepático da hidroquinona via CYP2E1. A monitorização biológica possui relevância nas situações de exposições ocupacionais. Para tal, utiliza-se o fenol urinário, considerado bioindicador de exposição a este composto. O objetivo do presente trabalho foi validar uma técnica de extração líquidolíquido para quantificar ofenol urinário, por meio da cromatografia em fase gasosa com detetor de ionização por chama (CG/DIC) em urina de trabalhadores expostos ao fenol em fundições. O método mostrou-se linear de 5 a $200 \mu \mathrm{g} / \mathrm{mL}$; coeficiente de regressão linear $\left(r^{2}\right)$ de 0,999; limites de deteç̧ão e quantificação 2,0 e 5,0 $\mu \mathrm{g} / \mathrm{mL}$, respectivamente; precisão intra-ensaio entre 4,5 e 8,9\% e interensaio entre 5,7 e 14,2\%, exatidão entre 6,2 e 11,9\% e recuperação superior a 87\%. O método demonstrou ser simples e rápido. Amostras provenientes de trabalhadores expostos ao fenol foram analisadas comprovando a aplicação da técnica na monitorização biológica.
\end{abstract}

\author{
Unitermos \\ - Fenol \\ - Cromatografia em fase \\ gasosa \\ - Monitorização biológica
}

\section{INTRODUÇÃO}

A utilização de resinas fenólicas na indústria de fundição data da década de 1950, quando foram amplamente empregadas em substituição aos cimentos naturais, tais como a bentonita para a confecção de moldes em área de macharia ou preparo de molde (Balmgärtel, 1984).
O processo usado na manufatura de moldes requer materiais moldantes, ligantes ou aglutinantes, compactantes e aceleradores de enrijecimento, dentre eles o fenol, com intuito de manter a estabilidade e a resistência à compressão (Mirer, 1998).

Neste contexto, o fenol é um composto da classe dos hidrocarbonetos aromáticos monossubstituídos, obtido naturalmente a partir do alcatrão da hulha e sendo 
freqüentemente utilizado como desinfetante industrial, na manufatura de nylons, bem como na indústria de petróleo. Apresenta-se no estado sólido, com aspecto cristalino e coloração fracamente rósea ou branca, odor acre, higroscópico, moderadamente volátil à temperatura ambiente, fracamente ácido e ionizado por reações eletrofílicas e de oxidação (Hee, Shane, 1993; WHO, 1994; Stork, 1997; Hodgson, Levi, 1997; OSHA, 2001; U. S. EPA, 2002; CHEMINFO, 2003).

Em exposições agudas o fenol apresenta toxicidade de moderada à alta. Em humanos, a dose letal (DL) é estimada em $70 \mathrm{mg} / \mathrm{kg}$ e a $\mathrm{DL}_{50}$ em ratos e coelhos é de $340 \mathrm{mg} / \mathrm{kg}$ e $850 \mathrm{mg} / \mathrm{kg}$, respectivamente. Estudos clínicos dermatológicos afirmam que o fenol é agressivo à pele e é utilizado em processos de peeling. Em exposições ocupacionais, o fenol pode levar a rash cutâneo, com o aparecimento de lesões eritematosas e necrose tecidual (WHO, 1994; Zimerson, 1997; Owen, Beck, 2001; U.S. EPA, 2002; CHEMINFO, 2003).

O fenol é rapidamente absorvido, após exposição por via inalatória, dérmica e oral, sendo distribuído por todos os tecidos. É biotransformado no fígado sofrendo reações de fase I e II e seus produtos de biotransformação podem ser eliminados a partir de conjugações com sulfato, glutationa e ácido glicurônico, principalmente via P4502E1 (CYP2E1). Além disso, pode sofrer ação na medula por mieloperoxidases liberando quinonas reativas, que seriam, via NQO1 (quinona oxidorredutase), reduzidas novamente a hidroquinona. Tal situação metabólica poderia aumentar o estresse oxidativo e posteriormente alterar o crescimento e diferenciação celular no compartimento medular (McDonald et al., 2001).

O fenol é eliminado pela urina, fezes, saliva e suor, sendo a primeira a principal via de excreção. Seu tempo de meia-vida biológica é de, em média, 12 horas (CHEMINFO, 1994).

Estudos in vivo e in vitro evidenciam a presença de ligações covalentes entre o fenol e proteínas plasmáticas, além de tecidos. Alguns produtos de biotransformação podem, também, ligarem-se às proteínas pelo fato de que o fenol e seus produtos de biotransformação, possuindo caráter eletrofílico, teriam afinidade com sítios nucleofílicos de grupamentos $\mathrm{N} \cdot \mathrm{O} \cdot \mathrm{ou} \mathrm{S} \cdot$ pertencentes a estruturas protéicas e/ou ao material genético (Kanerva et al., 1994; WHO, 1994; Snyder, 2004).

Dessa forma, a monitorização biológica - mensurações periódicas de biomarcadores, que podem ser definidos como "substâncias exógenas, metabólitos ou produtos da interação entre xenobióticos e algum alvo celular que é quantificado num compartimento do organismo" - possui papel relevante e complementar nas informações sobre situações de exposições laborais (Mutti, 1999; Vainio, 1998; Viau, 2002).

Para tal, a presença de fenol na urina é considerada indicador biológico da exposição ocupacional ao composto, sendo quantificado de acordo com a legislação específica (NR-7), em função da concentração da creatinina urinária (Tabela I) (Brasil, 1994).

No que se refere à metodologia utilizada na determinação biológica do fenol urinário vários autores sugerem técnicas analíticas tais como CG/DIC (cromatografia gasosa com detetor de ionização por chama), CLAE (cromatografia líquida de alto desempenho), CG/EM (cromatografia gasosa acoplada à espectrometria de massa), CL/EM (cromatografia líquida acoplada à espectrometria de massa) e espectrofotometria UV/VIS (Yamaguci, Hayashi, 1977; Ong et al., 1989; Mutti, 1999; Georgieva et al., 2002; Laurens et al., 2002; Boatto et al., 2004; Manini et al., 2004).

O presente trabalho teve por objetivo validar um método analítico para determinar fenol na urina, utilizando-se a técnica de cromatografia em fase gasosa com detetor de ionização por chama (CG/DIC) com a finalidade de sua aplicação na monitorização biológica de trabalhadores possivelmente expostos ao fenol em material à base de resinas fenólicas.

\section{MATERIAL E MÉTODOS}

\section{Soluções-padrão}

Foram preparadas soluções padrão de fenol e nitrobenzeno, utilizado como padrão interno, (PI) em metanol nas concentrações de $1000 \mu \mathrm{g} / \mathrm{mL}$, obtidos das empresas Riedel-de-Haën ${ }^{\circledR}$ e Androl Produtos Químicos ${ }^{\circledR}$, respectivamente.

TABELA I - Parâmetros da NR-7 * relativos à exposição ocupacional ao fenol

\begin{tabular}{lccccc}
\hline Material & IndicadorBiológico -IB & VR & IBMP & MétodoAnalítico & Amostragem \\
\hline Urina & Fenol & $20 \mathrm{mg} / \mathrm{g}$ creat. & $250 \mathrm{mg} / \mathrm{g}$ creat. & CG & FJ \\
\hline
\end{tabular}

* VR - Valor de referência, IBMP - Índice biológico máximo permitido, CG - Cromatografia gasosa, FJ - Final de jornada, exceto $1^{\text {a }}$ dia da semana, creat. - creatinina. (Brasil, NR - 7, 1994) 


\section{Amostras de trabalhadores expostos}

Amostras provenientes de 10 (dez) trabalhadores de indústria de fundição, 7 (sete) em contato com materiais à base de resinas fenólicas na área de macharia e 3(três) considerados controles não expostos, que trabalhavam na área de montagem e embalagem das peças metálicas, foram coletadas como preconizado pela Legislação (NR-7) vigente, de acordo com o projeto de pesquisa aprovado pelo Comitê de Ética em Pesquisa da FCF/USP (Ofício CEP n ${ }^{\circ}$ 077/2004) e analisadas no Laboratório de Pesquisas Toxicológicas, da Universidade de São Paulo.

\section{Preparo das amostras}

Para a padronização do método analítico foi utilizado um pool de urina de voluntários homens e mulheres sadios, acadêmicos da Universidade de São Paulo, que declararam não terem sido expostos ocupacionalmente a compostos fenólicos.

Além da exposição ocupacional, o fenol urinário pode ser proveniente de outras fontes, tais como substâncias presentes em alimentos e no ambiente (Scalbert, Williamson, 2000). Assim, com a finalidade de minimizar estas possibilidades, um pool de urina foi tratado com ácido clorídrico $37 \%$, na concentração de $10 \%$ em relação à urina, mantendo-se sob aquecimento até total evaporação. Esta urina foi considerada como controle negativo ou branco após ter sido reconstituída com água mili- $\mathrm{Q}^{\circledR}$.

\section{Otimização das condições cromatográficas}

Para melhor resolução cromatográfica do fenol foram estabelecidas as condições cromatográficas para a realização do experimento analítico.

Foi utilizado equipamento de cromatografia em fase gasosa Hewlett-Packard ${ }^{\circledR} 6890$ equipado com detector de ionização por chama (CG/DIC) nas seguintes condições: razão de divisão no injetor (1:20); coluna megabore de sílica fundida $100 \%$ de polidimetilsiloxano com as dimensões de $15 \mathrm{~m} \times 0,53 \mathrm{~mm} \times 1,50 \mu \mathrm{m}$ (HP-1); temperatura do injetor $200{ }^{\circ} \mathrm{C}$; gás de arraste, nitrogênio a uma vazão de $50 \mathrm{~mL} / \mathrm{min}$; programação da temperatura do forno: $60^{\circ} \mathrm{C}$ ( $2 \mathrm{~min}$ ), $10^{\circ} \mathrm{C} / \mathrm{min}$ até $120^{\circ} \mathrm{C}$ e $120^{\circ} \mathrm{C}$ por $2 \mathrm{~min}$.

\section{Método proposto para determinação do fenol em urina}

Em um tubo de vidro esmerilhado foram colocados $5,0 \mathrm{~mL}$ de urina controle negativo, $50 \mu \mathrm{L}$ do padrão interno, volumes relativos às concentrações desejadas do padrão analítico (a partir da solução-mãe de fenol $1000 \mu \mathrm{g} / \mathrm{mL}$ ) e $1,0 \mathrm{~mL}$ de ácido clorídrico $37 \%$. A mistura foi submetida a aquecimento em estufa a $95{ }^{\circ} \mathrm{C}$ por $1 \mathrm{~h}$ e $30 \mathrm{~min}$. Após resfriamento, foi adicionada à mistura $5,0 \mathrm{~mL}$ de éter etílico e $1,0 \mathrm{~g}$ de $\mathrm{NaCl}$. A mistura foi agitada em mesa agitadora, por 10 minutos e foram coletados $3 \mathrm{~mL}$ da fase orgânica e $2,0 \mu \mathrm{L}$ da mesma foram injetados no cromatográfo (CG-DIC).

\section{Determinação da densidade e da creatinina urinária}

As amostras de urina foram corrigidas e ajustadas pela medição da densidade e da determinação da creatinina urinária. A densidade foi medida com auxílio de um urodensímetro e os teores de creatinina foram determinados por método espectrofotométrico usando-se um Kit Labtest $^{\circledR}$.

\section{Validação do método}

As condições de preparação das amostras juntamente com a etapa de análise cromatográfica foram validadas para assegurar a qualidade, confiabilidade e aplicabilidade do método.

A validação foi implementada pelo estabelecimento dos valores de limite de detecção, limite de quantificação, linearidade, precisão intra e inter-ensaio, exatidão, recuperação e estabilidade do analito na matriz.

\section{Limites de detecção e de quantificação}

O limite de detecção (LD) foi determinado por meio de diluições sucessivas da amostra adicionada de fenol até a menor concentração detectável, na qual foi possível obter picos integrados pelo equipamento na relação de 3 vezes a relação sinal/ruído, nas condições cromatográficas otimizadas, em seis replicatas e coeficiente de variação menor que 20\% (Causon, 1997; Chasin et al., 1998; Queiroz et al., 2001).

O limite de quantificação (LQ) do método foi avaliado por meio da análise de amostra adicionada de concentrações decrescentes do composto até a menor concentração determinável com precisão e exatidão aceitáveis e com coeficiente de variação (CV) menor ou igual a $10 \%$. Para a realização das análises foi usado o procedimento descrito acima ( $\mathrm{n}=6)$ (Causon, 1997; Chasin et al., 1998).

\section{Linearidade}

Foram utilizadas as seguintes concentrações: 5, 10, $50,100,150$ e $200 \mu \mathrm{g} / \mathrm{mL}$ de fenol em urina, analisadas 
em triplicata/concentração, juntamente com $50 \mu \mathrm{g} / \mathrm{mL}$ de padrão interno.

A linearidade da curva foi avaliada por meio da equação da reta e do coeficiente de determinação $\left(\mathrm{r}^{2}\right)$ da curva, o qual deve ser superior a 0,98 (Causon, 1997; Chasin et al., 1998; Queiroz et al., 2001; Brasil, 2003).

\section{Precisão intra e inter-ensaio}

As precisões intra e inter-ensaio foram determinadas por meio da extração em sextuplicata de amostras fortificadas nas concentrações urinárias de 15,85 e $150 \mu \mathrm{g} / \mathrm{mL}$ analisadas no dia e em três dias consecutivos. A precisão foi calculada indiretamente, por meio da imprecisão, dada pelo coeficiente de variação $(\mathrm{CV})$ das áreas relativas obtidas, sendo que este não deve ser superior a 15\% (Causon, 1997; Chasin et al., 1998; Queiroz et al., 2001).

\section{Exatidão}

Para o estudo da exatidão, foi determinada a concentração de fenol presente em amostras consideradas como referência negativa, analisando-se o pool de amostras de urina obtidas de indivíduos controle negativo nas concentrações de 15,85 e $150 \mu \mathrm{g} / \mathrm{mL}$ em sextuplicata. A inexatidão foi calculada pela tendenciosidade (bias) de acordo com a equação:

Inexatidão $(\%)=\frac{\text { Conc. Obtida }- \text { Conc. Esperada x } 100}{\text { Conc. Esperada }}$

A exatidão não deve ser superior a $15 \%$ (Chasin et al., 1998; Queiroz et al., 2001; Brasil, 2003).

\section{Recuperação}

Com objetivo de se avaliar a eficiência do método de extração da amostra, após esta ter sido submetida à hidrólise ácida, conforme descrito acima, e, verificaremse possíveis alterações na determinação, amostras identificadas como brancos foram analisadas. Por comparação da resposta quando a adição foi feita antes da extração versus a resposta quando a adição foi realizada após o procedimento de extração, obteve-se a porcentagem do analito recuperado, posteriormente à hidrolise ácida (Chasin et al., 1998; Causon, 1997; Brasil, 2003).

\section{Estabilidade do analito}

A estabilidade do analito foi determinada pela quantificação do fenol adicionado na urina numa concen- tração de $50 \mu \mathrm{g} / \mathrm{mL}$. Alíquotas de $5 \mathrm{~mL}$ foram armazenadas $(n=3)$ em geladeira a $4{ }^{\circ} \mathrm{C}$ e em congelador a $\left(-20{ }^{\circ} \mathrm{C}\right)$. A estabilidade do analito foi calculada por meio do coeficiente de variação $(\mathrm{CV})$ das áreas dos picos relativos às analises realizadas após 6, 12, 24 e 48 horas. Os resultados foram comparados com aqueles obtidos nas análises do tempo zero (Causon, 1997).

\section{RESULTADOS}

A Figura 1 apresenta cromatogramas obtidos na análise de amostras de urina adicionada de $50 \mu \mathrm{g} / \mathrm{mL}$ de fenol, de trabalhador exposto e branco. A análise revela a presença de fenol na concentração de $38,1 \mathrm{mg} / \mathrm{g}$ creatinina.

A curva de calibração demonstrou ser linear (5 $200 \mu \mathrm{g} / \mathrm{mL}$ ). A equação de regressão linear e o coeficiente de determinação foram, respectivamente: $\mathrm{y}=0,0791 \mathrm{x}+$ 0,0558 e $\mathrm{r}^{2}=0,999$.

Os parâmetros de validação do método (LD, LQ, precisão intra e inter-ensaio, exatidão e recuperação) para determinação do fenol urinário estão dispostos na Tabela II.

\section{Estabilidade}

Os resultados do estudo da estabilidade do fenol em amostras de urina, adicionadas na concentração de $50 \mu \mathrm{g} / \mathrm{mL}$, são indicados nas Tabelas III e IV, referindose, respectivamente, à estabilidade do analito, em alíquotas de $5 \mathrm{~mL}$ de urina armazenadas $(\mathrm{n}=3)$ em geladeira a $4{ }^{\circ} \mathrm{C}$ e em congelador a $-20{ }^{\circ} \mathrm{C}$, após $6,12,24$ e 48 horas. A concentração obtida, corresponde à média da determinação realizada em triplicata da alíquota analisada.

As amostras de 10 (dez) trabalhadores, 3 (três) delas consideradas controles provenientes de indivíduos não expostos ao fenol, bem como 7 (sete) delas consideradas como sujeitos expostos, foram analisadas apresentando valores entre 7,7 - 38,1 $\mathrm{mg} / \mathrm{g}$ creatinina. Os resultados das análises, por trabalhador, estão dispostos na Tabela V.

\section{DISCUSSÃO}

Segundo a NR-7, fenol urinário é considerado indicador biológico de exposição ocupacional ao fenol. Este composto, apesar de ser encontrado na urina, originário de outras vias metabólicas (proveniente de alimentos ou de exposições a compostos que possuem o fenol como produto de biotransformação), é utilizado na monitorização biológica com valor de referência conhecido (Tabela I) e considerado para a correta avaliação da exposição ocupacional (Brasil, 1994). 


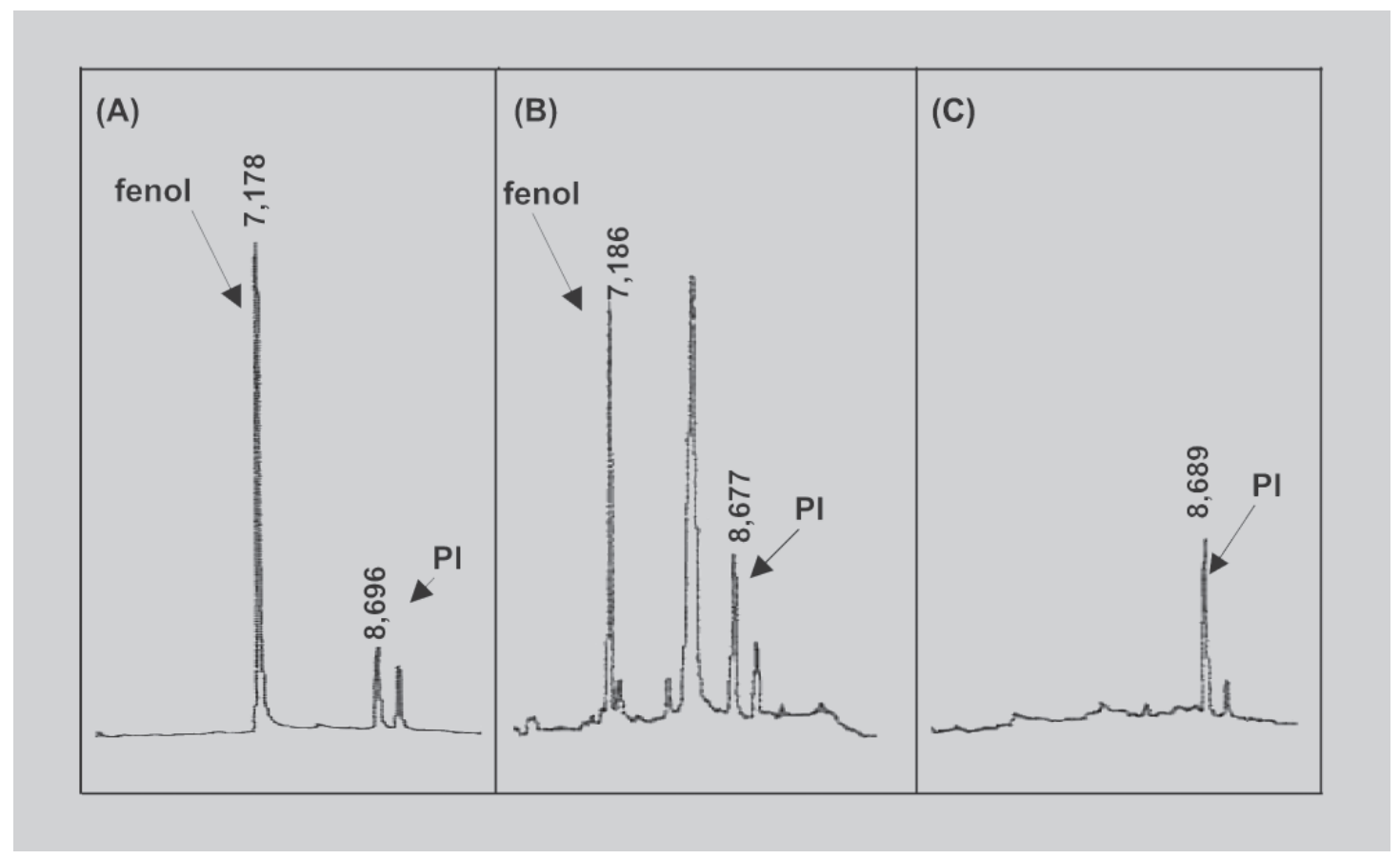

FIGURA 1 - Perfil cromatográfico obtido de amostras: (A) adicionada de fenol na concentração de $50 \mu \mathrm{g} / \mathrm{mL}$; (B) amostra de trabalhador exposto ao fenol na concentração de $38,1 \mathrm{mg} / \mathrm{g}$ creatinina e (C) amostra branco de urina. (PI padrão interno).

Alguns métodos para determinação de fenol em amostras biológicas têm sido relatados na literatura científica, utilizando-se técnica de espectrofotometria UV/VIS (Yamaguci, Hayashi, 1977), cromatografia líquida de alta eficiência (CLAE) (Mutti, 1999), cromatografia líquida de alta eficiência acoplada à espectrometria de massas (CL/EM) (Manini et al., 2004), cromatografia em fase gasosa com detetor de ionização por chama (CG/DIC) (Ong et al., 1989), cromatografia em fase gasosa acoplada à espectrometria de massas (CG/EM) (Laurens, 2002; Boatto et al., 2004). Resultados obtidos com esses métodos têm demonstrado a possibilidade de utilizá-los para determinação de fenol urinário em trabalhadores expostos em ambiente ocupacional.

A legislação brasileira recomenda a determinação do fenol urinário na monitorização biológica por método de cromatografia em fase gasosa com detetor de ionização por chama, de acordo com a Tabela I (Brasil, 1994).

Outras entidades internacionais, tais como a American Conference of Governmental Industrial Hygienists (ACGIH), o National Institute for Occupational Safety and Health (NIOSH), bem como a Organization for Safety and Health Administration (OSHA) preconizam a quantificação de fenol urinário, como indicador biológico da exposição ao fenol (Niosh, 1994; Osha, 2001; Acgih, 2005).
TABELA II - Parâmetros de confiança na validação do método de determinação de fenol urinário

\begin{tabular}{lc}
\hline Parâmetro & Fenol em urina \\
\hline $\mathrm{LD}(\mu \mathrm{g} / \mathrm{mL})$ & 2,0 \\
$\mathrm{LQ}(\mu \mathrm{g} / \mathrm{mL})$ & 5,0 \\
Precisão intra-ensaio $(\mathrm{CV}, \%)$ & \\
$\mathrm{C}_{1}$ & 8,9 \\
$\mathrm{C}_{2}$ & 4,8 \\
$\mathrm{C}_{3}$ & 4,5 \\
Precisão inter-ensaio $(\mathrm{CV}, \%)$ & \\
$\mathrm{C}_{1}$ & 14,2 \\
$\mathrm{C}_{2}$ & 5,7 \\
$\mathrm{C}_{3}$ & 8,9 \\
Recuperação $(\%)_{\mathrm{C}_{1}}$ \\
$\mathrm{C}_{2}$ & 95,7 \\
$\mathrm{C}_{3}$ & 87,9 \\
Inexatidão $(\mathrm{CV}, \%)$ & 96,2 \\
$\mathrm{C}_{1}$ & \\
$\mathrm{C}_{2}$ & 6,2 \\
$\mathrm{C}_{3}$ & 11,3 \\
\hline
\end{tabular}

LD: Limite de detecção; LQ: Limite de quantificação; $\mathrm{C}_{1}=15 \mu \mathrm{g} / \mathrm{mL} ; \mathrm{C}_{2}=85 \mu \mathrm{g} / \mathrm{mL} ; \mathrm{C}_{3}=150 \mu \mathrm{g} / \mathrm{mL}$; $\mathrm{CV}$ : Coeficiente de variação. 
TABELA III - Estudo da estabilidade do fenol em amostras de urina $(50 \mu \mathrm{g} / \mathrm{mL})$, mantidas em geladeira a $4{ }^{\circ} \mathrm{C}$

\begin{tabular}{cc}
\hline Tempo (horas) & Concentração $(\mu \mathrm{g} / \mathrm{mL})$ \\
\hline $0 *$ & 100 \\
$6 *$ & 46,2 \\
$12 *$ & 50,3 \\
$24 *$ & 54,3 \\
$48^{*}$ & 45,9 \\
\hline
\end{tabular}

*n: número de amostras analisadas $=3$.

TABELA IV - Estudo da estabilidade do fenol em amostras de urina $(50 \mu \mathrm{g} / \mathrm{mL})$, mantidas em freezer $\mathrm{a}-20{ }^{\circ} \mathrm{C}$

\begin{tabular}{cc}
\hline Tempo (horas) & Concentração $(\mu \mathrm{g} / \mathrm{mL})$ \\
\hline $0 *$ & 50 \\
$6 *$ & 49,3 \\
$12^{*}$ & 48,5 \\
$24 *$ & 48,2 \\
$48^{*}$ & 49,3 \\
\hline
\end{tabular}

*n: número de amostras analisadas $=3$.

TABELA V - Resultados das determinações de fenol urinário em trabalhadores não-expostos e expostos ocupacionalmente ao fenol

\begin{tabular}{lc}
\hline Amostra & Concentração (mg/g creatinina) \\
\hline Controle 1 & N.D. \\
Controle 2 & 7,7 \\
Controle 3 & 6,9 \\
Amostra 1 & 9,7 \\
Amostra 2 & 14,7 \\
Amostra 3 & 38,1 \\
Amostra 4 & 10,5 \\
Amostra 5 & 26,0 \\
Amostra 6 & 30,3 \\
Amostra 7 & 34,7 \\
\hline
\end{tabular}

Observação: (N.D.) = abaixo do LQ $(5,0 \mathrm{mg} / \mathrm{mL})$.

No presente trabalho, a cromatografia em fase gasosa com detetor de ionização por chama foi utilizada como técnica para a quantificação de fenol urinário. O método, baseado na extração líquido-líquido, demonstrou ser procedimento prático e rápido, em comparação a métodos que utilizam extração em fase sólida (SPE) (Manini et al., 2004) ou hidrólise enzimática (Adlard et al., 1981). O método utilizado neste trabalho permite a análise de 40 amostras diárias, oferecendo mais agili- dade na determinação analítica e comparando-se ao método similar validado por Ong et al. (1989), devido a modificações na coluna empregada e na programação na rampa analítica, com tempo de desenvolvimento de 10 minutos, em oposição aos 15 minutos observados no trabalho do autor.

No estudo da especificidade foi observada boa separação cromatográfica, os estudos de precisão intra e interensaios, com $\mathrm{CV}<15 \%$, demonstraram que alíquotas de $5 \mathrm{~mL}$ de urina para a determinação de fenol urinário são suficientes. Além disso, a utilização de éter etílico $(5 \mathrm{~mL})$ para a extração do fenol da fase aquosa demonstrou ser eficaz na técnica empregada.

A temperatura da estufa de $95^{\circ} \mathrm{C}$ não promoveu degradação importante do fenol e seu padrão interno, provavelmente pelo uso de tubo com tampa esmerilhada. Este permitiu equilíbrio entre a pressão de vapor $(\mathrm{pV})$ do solvente e a do ambiente tamponado. Após o aquecimento os tubos eram acondicionados em recipiente contendo gelo para oferecer menor influência na evaporação do fenol.

A adição de $\mathrm{NaCl}$ foi importante para promover o efeito "salting out", em que a adição do sal diminui a ligação do hidrogênio da água com o analito e, assim, a água é “seqüestrada " pela hidratação do sal (Ong et al., 1989).

O nitrobenzeno foi escolhido como padrão interno devido à semelhança estrutural em relação ao fenol, não ser encontrado normalmente na urina, ter ponto de ebulição relativamente próximo ao do fenol e tempo de eluição bastante satisfatório nas condições cromatográficas padronizadas. As duas substâncias são detectadas em menos de dez minutos, permitindo análise rápida (Ong et al., 1989; Niosh, 1994).

A técnica analítica proposta demonstrou ser altamente precisa e exata com o emprego do padrão interno nitrobenzeno. Valores adequados de sensibilidade e linearidade também foram obtidos para o analito. No parâmetro de recuperação a técnica empregada apresentou-se satisfatória demonstrando, por meio dos valores encontrados, perda aceitável frente às condições propostas (recuperação superior a $87 \%$ para o fenol). O coeficiente de determinação $\left(\mathrm{r}^{2}\right)$ foi superior a 0,99 dentro da faixa de interesse de $5-200 \mu \mathrm{g} / \mathrm{mL}$, sendo, portanto, apropriado (Causon, 1997; Chasin et al., 1998; Queiroz et al., 2001; Brasil, 2003).

A estabilidade foi avaliada segundo duas formas de conservação de amostras de urina: geladeira e freezer. As respectivas análises de estabilidade demonstraram que o método é estável nas condições preconizadas pela legislação em vigor e, no caso de transporte das amostras, estas podem ser acondicionadas em caixa de isopor com manu- 
tenção, por $48 \mathrm{~h}$, sem alteração significativa na perda do analito (Adlard et al., 1981).

O NIOSH considera como valor de referência de fenol urinário o intervalo entre $4,5-20,7 \mathrm{mg} / \mathrm{g}$ creatinina. Já a legislação brasileira preconiza VR (valor de referência) de $20 \mathrm{mg} / \mathrm{g}$ creatinina e Índice Biológico Máximo Permitido (IBMP) de $250 \mathrm{mg} / \mathrm{g}$ creatinina. Assim, valores de fenol urinário em amostras dos trabalhadores indicam que os indivíduos expostos, em indústria de fundição com preparo de materiais à base de resinas fenólicas, encontram-se abaixo do valor-limite considerado seguro para a saúde e bem-estar do indivíduo no ambiente de trabalho.

\section{CONCLUSÕES}

O método validado para determinação de fenol urinário por cromatografia em fase gasosa com detecção de ionização por chama demonstrou ser simples, rápido, eficiente, podendo ser facilmente aplicado na monitorização biológica de indivíduos expostos ao fenol.

O método validado pode ser usado na determinação de fenol urinário para monitorização biológica da exposição ocupacional.

\section{ABSTRACT}

Determination of urinary phenol by gas chromatography in workers using phenolic resins in foundries

Phenol is used as an industrial chemical, disinfectant agent, in the preparation of phenolic resins and paint pigments. When in solid state, it shows a light pink color, ocre odor, and is hygroscopic. In acute occupational exposure, the compound can produce erythemic injuries and burn sensation and, chronically, affect the cellular maturation of bone marrow due the free quinones and 1,4-benzoquinone, deriving from hepatic metabolism of the hydroquinone by P450 isozyme (CYP2E1). The biological monitoring is important in occupational exposure situations. So, urinary phenol, considered the biological indicator of phenol exposure has to be determined. The aim of this work was to validate a method for urinary phenol quantification, using liquidliquid extraction. Gas chromatography with flame ionization detector (GC/FID) was used in this method. The analytical procedure showed linearity in the dynamic range of the assay from $5-200 \mu \mathrm{g} / \mathrm{mL}$. The limits of detection (LOD) and quantification ( $L O Q)$ were: 2.0 and $5.0 \mu \mathrm{g} / \mathrm{mL}$, respectively. Intra-assay precision coefficient was between $4.5-8.9 \%$ and the inter-assay precision coefficient was between 5.7 $14.2 \%$. The accuracy coefficient was between $6.2-$ $11.9 \%$. Recovery values were higher than $87 \%$. The coefficient of correlation was 0.999. The method is simple, rapid and efficient. Urine samples from workers exposed to phenolic resins were analyzed to show the method application in biological monitoring.

UNITERMS: Phenol. Gas chromatography. Biological monitoring.

\section{AGRADECIMENTOS}

Agradecemos aos Professores Maurício Yanamine e Ernani Pinto Júnior nos aspectos analíticos, à Fundacentro/SP e à CAPES/PROAP ao apoio financeiro e logística necessária para a realização do trabalho.

\section{REFERÊNCIAS BIBLIOGRÁFICAS}

AMERICAN CONFERENCE of GOVERMMENTA INDUSTRIAL HIGYENISTS. (ACGIH). $2005 T L V s^{\hat{a}}$ and BEIs ${ }^{\circledR}$ based on the documentation for threshold limit values for chemical substances, physical agents and biological exposure indices. Cincinnati: ACGIH, 2005. 219p.

ADLARD, E.R.; MILNE, P. E.; TINDLE, P. E. Determination of phenol by Gas Cromatography with enzymatic hydrolysis. Chromatographia, New York, v.14, p. $507,1981$.

BAUMGÄRTEL, I. Dangerous working substances in foundries - mold materials. Vienna: International Section for the Prevention of Occupational Accidents and Diseases in the Iron and Metal Manufacturing Industry of the International Social Security Association, 1984.43 p.

BOATTO, G.; NIEDDU, M.; CARLA, A.; PAU, A.; LORENZONI, S.; MANCONI, P.; SERRA, D. Determination of phenol and o-cresol by GC/MS in a fatal poisoning case. Forens. Sci. Int., Dublin,v. 139, p. $191-194,2004$

BRASIL. Ministério da Saúde. [ANVISA] - Agência Nacional de Vigilância Sanitária. Legislação. Resoluções. Resolução n 899, de 29 de maio de 2003. Anexo I: Guia para validação de métodos analíticos e bioanalíticos. 2003. Disponível em: http://www.anvisa.gov.br/legis/ resol/2003/re/899 03.htm. Acesso em: 13 out. 2004. 
BRASIL. Ministério do Trabalho e Emprego. NORMA REGULAMENTADORA $n^{\circ} 7$. Brasil: Ministério do Trabalho e Emprego,1994. Disponível em: http:// www.mte.gov.br/empregador/segsau/legislacao/normas/ conteudo/nr07/default.asp. Acesso em: 07 mar. 2005.

CAUSON, R. Validation of chromatographic methods in biomedical analyses - Viewpoint and discussion. $J$. Chromatogr. B., Amsterdam, v. 689, p. 175-180, 1997.

CHASIN, A.A.M.; NASCIMENTO, E. S.; NETO, L.M.R.; SIQUEIRA, M.E.P.B.; ANDRAUS, M.H.; SALVADORI, M.C.; FERNÍCOLA, N.A.G.; GORNI, R.; SALCEDO, S. Validação de métodos em análises toxicológicas: uma abordagem geral. Rev. Bras. Tox., São Paulo, v. 11, n.1, p. 1-6, 1999.

CHEMISTRY INFORMATION. (CHEMINFO) Phenol. Hamilton: Canadian Centre for Occupational Health and Safety, 2003. 19 p.

GEORGIEVA, T. MICHAILOVA, A. PANEV, T.; POPOV, T. Possible to control the health risk of petrochemical workers. Int. Arch. Occup. Environ. Health, Berlin, v. 75, S 21-S 26, 2002.

HEE, Q.; SHANE, S. Biological monitoring: an introduction. 1. ed. New York: Van Nostrand Reinhold, 1993. p. 471.

HODGSON, E.; LEVI, P. E., A textbook of modern toxicology. 2. ed. Connecticut: Stamford, 1997. p. 244 - 247.

KANERVA, L.; TARVAINEN, K.; PINOLA, A.; LEINO, T.; GRANLUND, H.; ESTLANDER, T.; JOLANKI, R.; FÖRSTRÖM, L. A single accidental exposure may result in a chemical burn, primary sensitization and allergic contact dermatitis. Contact Derm., Copenhagën, v. 31, p. 229-235, 1994.

LAURENS, J.B.; MBIANDA, X. Y.; SPIES, J. H.; UBBINK, J. B.; VERMAAK, W.J.H. Validated method for quantification of biomarkers for benzene and its alkylated analogues in urine. J. Chromatogr. B, Amsterdam, v. 774, p. 173-185, 2002.

MANINI, P; ANDREOLI, R; NIESSEN, W. M. A. Liquid cromatography - mass spectrometry in occupational toxicology: A novel approach to the study of biotransformation of industrial chemicals. J. Chromatogr. A, Amsterdam, v. 1058, p. 21-37, 2004.
MCDONALD, T. A; HOLLAND, N. T.; SKIBOLA, C.; DURAMAD, P.; SMITH, M. T. Hypothesis: Phenol and hydroquinone derived from diet and gastrointestinal flora activity are causal factors in leukemia. Leukemia, London, v. 15, p. 10-20, 2001.

MIRER, F. E. Fudiciones. In. STELLMANN, L. Enciclopedia de salud y securidad en el trabajo. 3. ed. Madrid: Oficina International del Trabajo - OIT Ministério del Trabajo Y Assuntos Sociales, 1998. v. II, p. 8215-22.

MUTTI, A. Biological monitoring in occupational and environmental toxicology. Toxicol. Lett., Amsterdam, v. 108 , p. 77-89, 1999

NATIONAL INSTITUTE FOR OCCUPATIONAL AND SAFETY HEALTH. (NIOSH). Manual of analitical methods - phenol and cresol isomers. 4. ed. Washington: NIOSH, 1994, 5 p. (Method 2546, Issue 1, dated 15 august 1994).

ONG, C. N.; LEE, B. L.; ONG, H. Y.; HENG, L. E. Determination of urinary phenol by acid hydrolysis and gas chromatography. J. Anal. Toxicol., Niles, v. 12, p. 159-161, 1989.

OCCUPATIONAL SAFETY AND HEALTH ADMINISTRATION. (OSHA). Phenol. International Chemical Safety Cards. Washington: OSHA, 2001, 5 p., (International Safety Cards, 70).

OWEN, C. M.; BECK, M. Occupational allergic contact dermatitis from phenol-formaldehyde resins. Contact Derm., Copenhagen, v. 45, p. 294-295, 2001.

QUEIROZ, S. C. N.; COLLINS, C. H.; JARDIM, I. S.F. Métodos de extração e/ou concentração de compostos encontrados em eluídos biológicos para posterior determinação cromatográfica. Quim. Nova., São Paulo, v. 1, p. $68-76,2001$.

SCALBERT, A.; WILLIANSOM, G. Dietary intake and bioavailability of polyphenols. J. Nutr., Rockville, v. 130, n. 8, suppll., 2000, S 2073-S 2085.

SNYDER, R. Xenobiotic metabolism and the mechanism(s) of benzene toxicity. Drug Met. Rev., Little Rock, v. 36, n. 4, p. 531-547, 2004. 
UNITED STATES - ATSDR. Department of Health and Human Services. Public Health Service. Agency for Toxic Substances and Disease Register. Toxicological profile for phenol. 1998. Disponível em: http://www. atsdr.cdc.gov/toxprofiles/phenol69.html. Acesso em: 14 fev. 2005.

VAINIO, H. Use of biomarkers - new frontiers in occupational toxicology and epidemiology. Toxicol. Lett., Amsterdam, v. 102-103, p. 581 - 589, 1998.

VIAU, C. Biological monitoring of exposure to mixtures. Toxicol. Lett., Amsterdam, v. 134, p. 9 -16, 2002.
WORLD HEALTH ORGANIZATION. (WHO). Phenol. International Programme on Chemical Safety. Geneva: WHO, 1994, 98 p., (Environmental Health Criteria, 412).

YAMAGUCI, Y.; HAYASHI, C. Determination of urinary total phenolic compounds with use of 4-aminoantipyrine: suggested screening test for hyperthyroidism and for catecholamine-producing tumor. Clin. Chem., Washington, v. 23, p. 2151-2154, 1977.

ZIMERSON, E.; BRUZE, M. Cross-reaction in patients with contact allergy to simple methylol phenols. Contact. Derm., Copenhagen, v. 37, n. 2, p. 82 - 86, 1997.

Recebido para publicação em 04 de maio de 2005. Aceito para publicação em 30 de janeiro de 2006. 